\title{
Electronic Polarization of 1H-Benzotriazole in Water: Ground and First Excited-State Dipole Moments
}

\author{
VALDEMIR LUDWIG, ${ }^{1}$ KALINE COUTINHO, ${ }^{2}$ \\ ANTONIO CARLOS BORIN, ${ }^{3}$ SYLVIO CANUTO ${ }^{1}$ \\ ${ }^{1}$ Instituto de Física, Universidade de São Paulo, CP 66318, 05315-970 São Paulo, Brazil \\ ${ }^{2}$ Universidade de Mogi das Cruzes, CP 411, 08701-970 Mogi das Cruzes, São Paulo, Brazil \\ ${ }^{3}$ Instituto de Química, Universidade de São Paulo, Av. Prof. Lineu Prestes, 748, \\ 05508-900 São Paulo, Brazil
}

Received 20 March 2003; accepted 29 April 2003

DOI 10.1002/qua.10688

\begin{abstract}
Sequential Monte Carlo and quantum mechanical calculations of the electronic polarization of the ground and first vertical excited states of $1 \mathrm{H}$-benzotriazole in water are made. Using statistically uncorrelated configurations and CASSCF ab initio calculations the increase in the ground and excited state dipole moments of $1 \mathrm{H}$ benzotriazole from gas phase to aqueous environment are obtained as $2.89 \pm 0.10$ and $2.75 \pm 0.11 \mathrm{D}$, leading to total moments of $6.89 \pm 0.10$ and $6.40 \pm 0.11 \mathrm{D}$, respectively. Structures are sampled using the statistical correlation interval obtained from the autocorrelation function of the energy and statistical convergence of the calculated dipole moments is shown. These water-polarized dipole moments of $1 \mathrm{H}$-benzotriazole are used to obtain the solvatochromic shift of the first absorption transition. The resulting theoretical blue shift of $570 \mathrm{~cm}^{-1}$ for the strong and characteristic $\pi \rightarrow \pi^{*}$ transition is in good agreement with the inferred experimental value of $\sim 700 \mathrm{~cm}^{-1}$. The use of the gas-phase values of the dipole moments are useful for the qualitative understanding of the shift but the polarization of the solvent is required to achieve quantitative agreement. (c) 2003 Wiley Periodicals, Inc. Int J Quantum Chem 95: 572-579, 2003
\end{abstract}

Key words: QM/MM method; solvent effects; dipole moments; benzotriazole

\section{Introduction}

$\mathbf{T}$ he interaction with a solvent leads to an electronic polarization of the solute [1]. Understanding this polarization is important for the the-

Correspondence to: S. Canuto; e-mail: canuto@if.usp.br oretical description of the changes in the spectroscopic properties of a reference molecule [2, 3]. It is known that the electronic polarization leads to an increase of the dipole moment and this has been the subject of great interest in recent years [2]. The increase in the ground-state dipole moment is crucial to understand the dielectric properties of liquids. Similarly, the proper description of the 


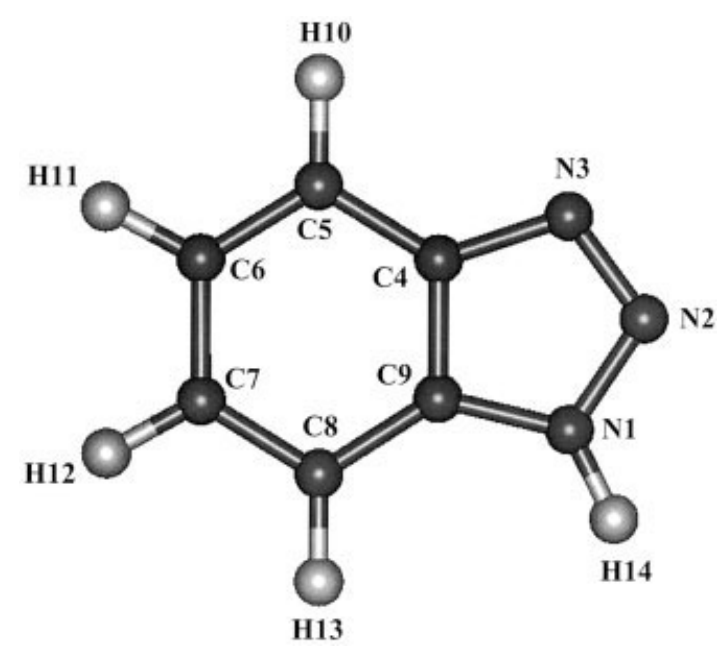

FIGURE 1. 1H-BZT. Atomic indices are used to define the simulation parameters given in Table $\mathrm{I}$.

electronic polarization is essential for classic force fields in molecular design [4]. A number of theoretical works have addressed this issue. A notorious case is the increase in the ground-state dipole moment of liquid water that has been subjected to numerous theoretical investigations [5-8]. Similar studies of the electronic polarization of organic molecules in water have appeared before [2]. Direct experimental measurement of the dipole moment in liquids is not possible. From the point of view of electronic absorption spectroscopy, the relative values of the dipole moment of the ground and excited states can be related to solvatochromic shifts [9]. It is expected that a decrease (increase) in the dipole moment upon excitation leads to a blue (red) shift. This rule implies, for instance, that $n \rightarrow \pi^{*}$ transitions are blue shifted. Similar reasoning, with consideration of the dipole polarizabilities, leads to the conclusion that if there is no change in the dipole moment, such as in the case of most $\pi \rightarrow \pi^{*}$ transitions, it will normally result in a red shift [10].

In this article we are interested in the electronic polarization of the ground and first excited states of $1 \mathrm{H}$-benzotriazole (1H-BZT). In fact, benzotriazole may exist in two tautomeric forms defined by the position of the $\mathrm{NH}$ group in the five-atom ring (see Fig. 1). The relative stability of the $1 \mathrm{H}$ and $2 \mathrm{H}$ tautomers have been studied both theoretically and experimentally by several groups [11-20]. There are recent experimental [17] indications that in the gas phase the $1 \mathrm{H}$ is more stable than the $2 \mathrm{H}$ by only ca. $1.2 \mathrm{kcal} / \mathrm{mol}$. It is also evident in several experiments that in the gas phase both tautomers may coexist [11-14]. However, in solution it is clear that the $1 \mathrm{H}$ tautomer is indeed more stable, in particular in the case of polar solvents $[13,15,17-20]$. This is likely to be an immediate consequence of the fact that the dipole moment of the $1 \mathrm{H}$ form is considerably larger [13] (by a factor close to 10) than the $2 \mathrm{H}$. There are indeed several experiments, including direct NMR measurements [19], indicating that in solution the $1 \mathrm{H}$ form is the most stable. UV-Vis experiments in different solvents [20] show that the absorption spectrum of benzotriazole is consistent with the $1 \mathrm{H}$ form for all solvents, including the nonpolar and nonprotic ones.

Experimental UV-Vis spectra of benzotriazole in different solvents [20] indicate that the characteristic strong $\pi \rightarrow \pi^{*}$ transition is blue shifted with respect to the transition in the gas phase. As it is normally expected that $\pi \rightarrow \pi^{*}$ transitions in solvents are red shifted from the gas phase or lowpolarity solvents, the theoretical investigation of this solvatochromic shift becomes relevant. The observed blue shift can, of course, be understood if there is a decrease in the dipole moment upon excitation.

For all the reasons considered above, the study of the ground- and excited-state dipole moment of $1 \mathrm{H}$ benzotriazole in the gas phase and in polar solvents is of great interest. In this article, we use the sequential Monte Carlo quantum mechanics (SMC/QM) methodology [21, 22] with ab initio CASSCF [23] calculations performed on structures generated by MC simulations to give reliable and consistent values for the dipole moments of both the ground and excited states of $1 \mathrm{H}-\mathrm{BZT}$ in liquid water. A liquid is statistical by nature and its structure does not correspond to a minimum energy configuration. Hence, the proper description of the liquid structure needs statistical procedures. Therefore, we first perform a MC statistical mechanics simulation of $1 \mathrm{H}-\mathrm{BZT}$ in water to generate the liquid structure of the solution. Subsequently, we use these structures of the solute-solvent to perform the QM CASSCF calculations. The size of the supermolecular structure that is extracted from the simulation for the subsequent QM calculations are selected on the basis of the corresponding solvation shells, leading to structures composed of the solute and all solvent molecules within a certain solvation shell. Here, we consider all the supermolecular structures up to the fourth solvation shells, at a distance of $12.2 \AA$ away from the center-of-mass of the solute $(1 \mathrm{H}-\mathrm{BZT})$. Instead of performing these costly QM calculations in all configurations generated by the simulation we use the autocorrelation 
LUDWIG ET AL.

TABLE I

Geometries and parameters of the Coulomb-Lennard-Jones potential of 1H-BZT and water molecules.

\begin{tabular}{|c|c|c|c|c|c|c|}
\hline & \multicolumn{3}{|c|}{ Coordinates $(x, y, z)$} & $q$ & $\varepsilon(\mathrm{kcal} / \mathrm{mol})$ & $\sigma(\AA ̊)$ \\
\hline \multicolumn{7}{|c|}{$1 \mathrm{H}-\mathrm{BZT}$} \\
\hline $\mathrm{N} 1$ & 1.3222 & -1.1091 & 0.0000 & -0.1300 & 0.170 & 3.250 \\
\hline $\mathrm{N} 2$ & 2.0662 & 0.0000 & 0.0000 & -0.1852 & 0.170 & 3.250 \\
\hline N3 & 1.3051 & 1.0213 & 0.0000 & -0.3836 & 0.170 & 3.250 \\
\hline $\mathrm{C} 4$ & 0.0000 & 0.5897 & 0.0000 & 0.4939 & 0.145 & 3.750 \\
\hline C5 & -1.2059 & 1.3179 & 0.0000 & -0.3475 & 0.070 & 3.550 \\
\hline C6 & -2.3796 & 0.5903 & 0.0000 & -0.0810 & 0.070 & 3.550 \\
\hline $\mathrm{C7}$ & -2.3680 & -0.8311 & 0.0000 & -0.0892 & 0.070 & 3.550 \\
\hline $\mathrm{C} 8$ & -1.1889 & -1.5528 & 0.0000 & -0.3126 & 0.070 & 3.550 \\
\hline $\mathrm{C9}$ & -0.0000 & -0.7997 & 0.0000 & 0.1441 & 0.145 & 3.750 \\
\hline $\mathrm{H} 10$ & -1.2006 & 2.3918 & 0.0000 & 0.1814 & 0.020 & 2.500 \\
\hline $\mathrm{H} 11$ & -3.3258 & 1.1006 & 0.0000 & 0.1115 & 0.020 & 2.500 \\
\hline $\mathrm{H} 12$ & -1.1863 & -2.6274 & 0.0000 & 0.1743 & 0.020 & 2.500 \\
\hline $\mathrm{H} 13$ & -3.3059 & -1.3572 & 0.0000 & 0.1209 & 0.020 & 2.500 \\
\hline $\mathrm{H} 14$ & 1.7738 & -1.9921 & 0.0000 & 0.3030 & 0.020 & 2.500 \\
\hline \multicolumn{7}{|l|}{ Water } \\
\hline $\mathrm{O}$ & 0.0000 & 0.0000 & 0.0000 & -0.8200 & 0.1550 & 3.1650 \\
\hline $\mathrm{H}$ & -0.5678 & -0.4652 & 0.0000 & 0.4100 & 0.0000 & 0.0000 \\
\hline $\mathrm{H}$ & -0.3486 & 0.8911 & 0.0000 & 0.4100 & 0.0000 & 0.0000 \\
\hline
\end{tabular}

See Figure 1 for definitions of the atomic indices.

function of the energy [21,22] to sample statistically relevant configurations. An important point in this issue is that statistical convergence is obtained (see below) by using this efficient sampling technique.

\section{Theoretical Methodology}

MC simulations are carried out employing standard procedures [24], including the Metropolis sampling technique and periodic boundary conditions using the minimum image method in a cubic box. The simulations are performed in the NVT ensemble. The total system consists of the 1H-BZT plus 700 water molecules at room temperature (298 $\mathrm{K})$ and density of $0.9966 \mathrm{~g} / \mathrm{cm}^{3}$. The intermolecular interactions are described by the standard Lennard-Jones plus Coulomb potentials with three parameters for each site $i\left(\varepsilon_{i}, \sigma_{i}\right.$, and $\left.q_{i}\right)$. For the water molecules we use the SPC potential [25]. The geometry of water is slightly modified, giving a slight increase in the classical dipole moment of water (2.31 D instead of the original 2.28 D). For 1H-BZT we use the OPLS [26] parameters $\left(\varepsilon_{i}\right.$ and $\left.\sigma_{i}\right)$ in the optimized geometry obtained by means of the CASSCF method [23], using the 6-31G $(d, p)$ basis set for carbon, nitrogen, and hydrogen atoms, assuming $C_{s}$ symmetry. The active spaces included all $\pi$, $\pi^{*}$ valence electrons and orbitals. The optimization was carried out with the MOLPRO [27] suite of programs. The QM calculated dipole moment of isolated $1 \mathrm{H}-\mathrm{BZT}$ is $4.0 \mathrm{D}$, in good agreement with the experimental value [16] of $4.3 \mathrm{D}$. The classic charges for 1H-BZT were then obtained from an electrostatic fit using CHELPG [28] in a single-point calculation using MP2/6-31+G(d), which gave a dipole of $4.13 \mathrm{D}$. Table I summarizes the results for the geometries and the parameters of the CoulombLennard-Jones potential used in the simulation. The calculated geometry is in good agreement with the experimental [29] result. This can also be seen by comparing the theoretical rotational constants (1191, 1686, and $4060 \mathrm{MHz}$ ) with the recent experimental results [16] $(1182,1676$, and $4008 \mathrm{MHz})$. The intermolecular interactions are spherically truncated within a center of mass separation larger than the cut-off radius, $r_{\mathrm{C}}=13.84 \AA$. Long-range corrections were calculated beyond this cut-off distance. The Lennard-Jones potential contribution is estimated assuming an uniform distribution $G(r) \approx 1$ after the cut-off radius and the electrostatic potential contribution is estimated with the reaction field method of the dipolar interaction. In the simulation the molecules are kept with rigid geometries. The initial configurations are generated randomly, considering the position and orientation of each mole- 
cule. A new configuration is generated after randomly attempt to translate in all Cartesian directions and rotate around a randomly chosen axis. The simulations consist of a thermalization phase of $26 \times 10^{6}$ MC steps, followed by an averaging stage of $35 \times 10^{6} \mathrm{MC}$ steps. To analyze the electronic structure of $1 \mathrm{H}-\mathrm{BZT}$ in liquid water we employ the S-MC/QM procedure [21, 22]. The QM calculations are performed on the supermolecular structures, generated by the MC simulations, composed of a central 1H-BZT molecule and all SPC point-charge water molecules within a particular solvation shell. The great advantage of the sequential procedure of the S-MC/QM is that all the important MC statistical information is available before running into the QM calculations [30-32]. This considerably reduces the number of supermolecular structures that will be submitted for the quantum mechanical calculations because the configurations are selected according to their statistical correlation, obtained from the autocorrelation function of the energy [30-33]. Using a correlation step of $7 \times 10^{5}$, we separate a total of 50 configurations, with less than $10 \%$ of statistical correlation. These 50 structures are used in the quantum mechanical calculations. On the other hand, this number of QM calculations is still tractable so that high-level statespecific (separate iterations for the ground and excited states) size-extensive CASSCF calculations can be used. The solvation shells were defined from the analysis of the radial distribution function. The electronic structure calculations of each supermolecular structure followed a well-established methodology employed by us in previous opportunities [34-38]. First, the CASSCF method was used to compute the reference function and dipole moments for the ground and first excited states using the same active space described above. Then, the CASSCF reference function was employed to compute the vertical excitation energy at the CASPT2 level [34, 39, 40]. Intruder states were removed using the level-shift technique (LS-CASPT2) [3941] with a level-shift parameter of 0.3 a. These calculations were performed with the MOLCAS-5.0 program package [42], using atomic natural orbitals (ANO-S) basis sets [43], with the contraction schemes $[4 s 3 p 2 d]$ for carbon and nitrogen atoms and $[2 s 1 p]$ for the hydrogens. The dipole moments reported are the results of a simple average over the QM CASSCF results for the 50 statistically uncorrelated supermolecular configurations. All simulations are performed with the DICE [44] MC statistical mechanics program.

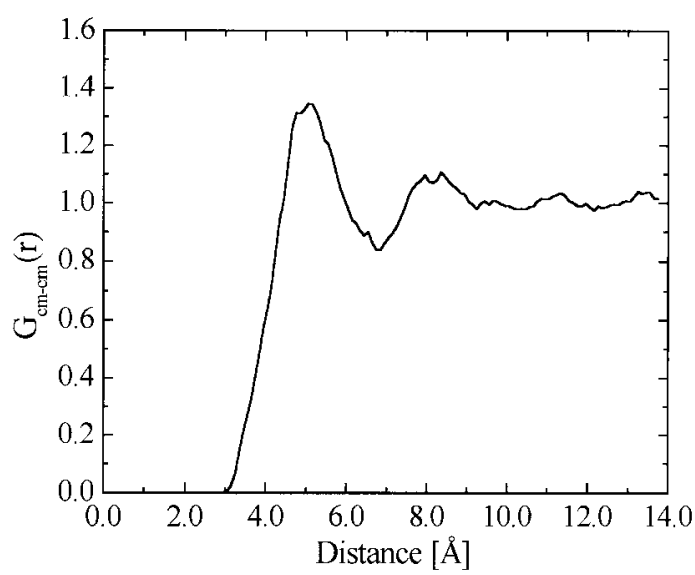

FIGURE 2. Calculated radial distribution function between the center-of-mass of $1 \mathrm{H}-\mathrm{BZT}$ and the center-ofmass of the water molecules.

Figure 2 shows the calculated radial distribution function of the center-of-mass of $1 \mathrm{H}-\mathrm{BZT}$ and the center-of-mass of water with a clear first solvation shell ending in $6.9 \AA$. The other structures are more difficult to discern and we selected the distances of 9.3, 10.3, and $12.2 \AA$. Spherical integration up to these distances give, respectively, 40, 107, 147, and 247 water molecules. For the first solvation shell, however, we found it preferable to use a minimumdistance solvation shell [30] instead of a spherical distribution, leading to 36 water molecules. We then separated supermolecular structures composed of the central 1H-BZT molecule and 36, 107, 147, and 247 SPC water molecules. These will be referred to as $1+36,1+107,1+147$, and $1+247$ SPC, respectively. Figure 3 shows one of the supermolecular structures used in the QM calculations for the first solvation shell $(1+36$ SPC). The best results obtained here use averages of $50 \mathrm{QM}$ calculations at the CASSCF level using $1+247$ SPC structures for both the ground- and excited-state dipole moments.

\section{Results and Discussion}

\section{CALCULATED DIPOLE MOMENTS}

Table II summarizes the results for the dipole moments of both the ground and excited states of 1H-BZT. For the isolated molecule the calculated vertical excited-state dipole moment is decreased with respect to the ground state by $0.35 \mathrm{D}$, leading to a total dipole moment of $3.65 \mathrm{D}$. This decrease in dipole moment upon vertical excitation is enough 


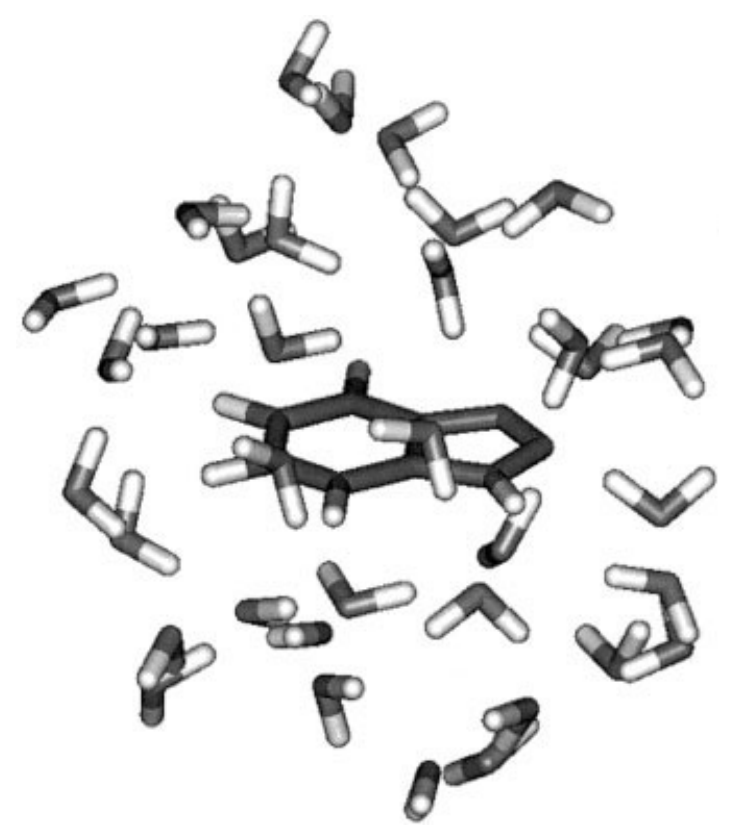

FIGURE 3. Illustration of one supermolecular structure generated by the simulation corresponding to the minimum-distance first solvation shell $(1 \mathrm{H}-\mathrm{BZT}+36 \mathrm{SPC}$ model).

to qualitatively understand the blue shift observed experimentally. However, it is important now to consider the solvent effect. Inclusion of the first solvation shell increases the ground-state dipole moment by as much as $2.15 \mathrm{D}$. Systematic consideration of the outer solvation shells leads to a converged value (with respect to the number of solvent molecules) of $6.89 \pm 0.10 \mathrm{D}$, where the uncertainty is the calculated statistical error. This result indicates a considerable polarization of the ground state, leading to an induced dipole moment of as much as $2.89 \pm 0.10 \mathrm{D}$. Similar results are obtained for the first $\pi \rightarrow \pi^{*}$ vertical excited state. Inclusion of the first solvation shell increases the excited-state dipole moment by 2.09 D. Consideration of the outer solvation shells leads to an increase of $2.75 \pm$ $0.11 \mathrm{D}$ and the total value of $6.40 \pm 0.11 \mathrm{D}$ for the dipole moment of the excited state. Analysis of the results shown in Table II indicates that the most important contribution comes from the first solvation shell but the outer shells also contribute and these dipole moments seem converged with respect to the size of the supermolecular structures. Statistical convergence will be considered below. The polarization of the solvent is stronger for the ground than for the first excited state. This makes the decrease of the dipole moment upon vertical excitation to change from $0.35 \mathrm{D}$ (gas phase) to 0.49 $\mathrm{D} \pm 0.21 \mathrm{D}$ (in water). Solvent effects in dipole moments are not possible to be obtained directly from experiment. This quantitative change will therefore be considered below to show a good numerical agreement with the observed blue shift of the absorption spectrum.

\section{SAMPLING AND STATISTICAL CONVERGENCE}

The question of sampling configurations is crucial for the efficiency of the QM/MM methodology and has been our concern before [21, 22, 32]. We analyzed the statistical correlation between the configurations generated and only those statistically relevant are used in the calculation of the average. The statistical correlation is obtained from the autocorrelation function of the energy [21, 32]. We have shown previously that the average over all successive configurations generated in the MC simulation give the same result as averaging over only a few statistically uncorrelated configurations [45]. This efficient way to sample configurations gives statistically converged results $[30,31,46]$. Here, we selected one configuration every $7 \times 10^{5} \mathrm{MC}$ steps and used them to perform the QM calculations. The

TABLE II

Calculated dipole moments (Debye) using state-specific CASSCF/6-31G(d,p) calculations of 1H-BZT in water solvent and calculated solvatochromic shift $\left(\mathrm{cm}^{-1}\right)$ obtained with Eq. (1).

\begin{tabular}{lccc}
\hline System & Ground state & Excited state & Solvatochromic shift \\
\hline 1H-BZT (gas phase) & 4.00 & 3.65 & 230 \\
$1 \mathrm{H}-\mathrm{BZT}+36$ SPC & $6.15 \pm 0.13$ & $5.74 \pm 0.16$ & 420 \\
$1 \mathrm{H}-\mathrm{BZT}+107$ SPC & $6.79 \pm 0.13$ & $6.28 \pm 0.15$ & 580 \\
$1 \mathrm{H}-\mathrm{BZT}+147$ SPC & $6.88 \pm 0.13$ & $6.36 \pm 0.16$ & 600 \\
$1 \mathrm{H}-\mathrm{BZT}+247$ SPC & $6.89 \pm 0.10$ & $6.40 \pm 0.11$ & 570
\end{tabular}

Results represent averages of over $50 \mathrm{QM}$ calculations. 

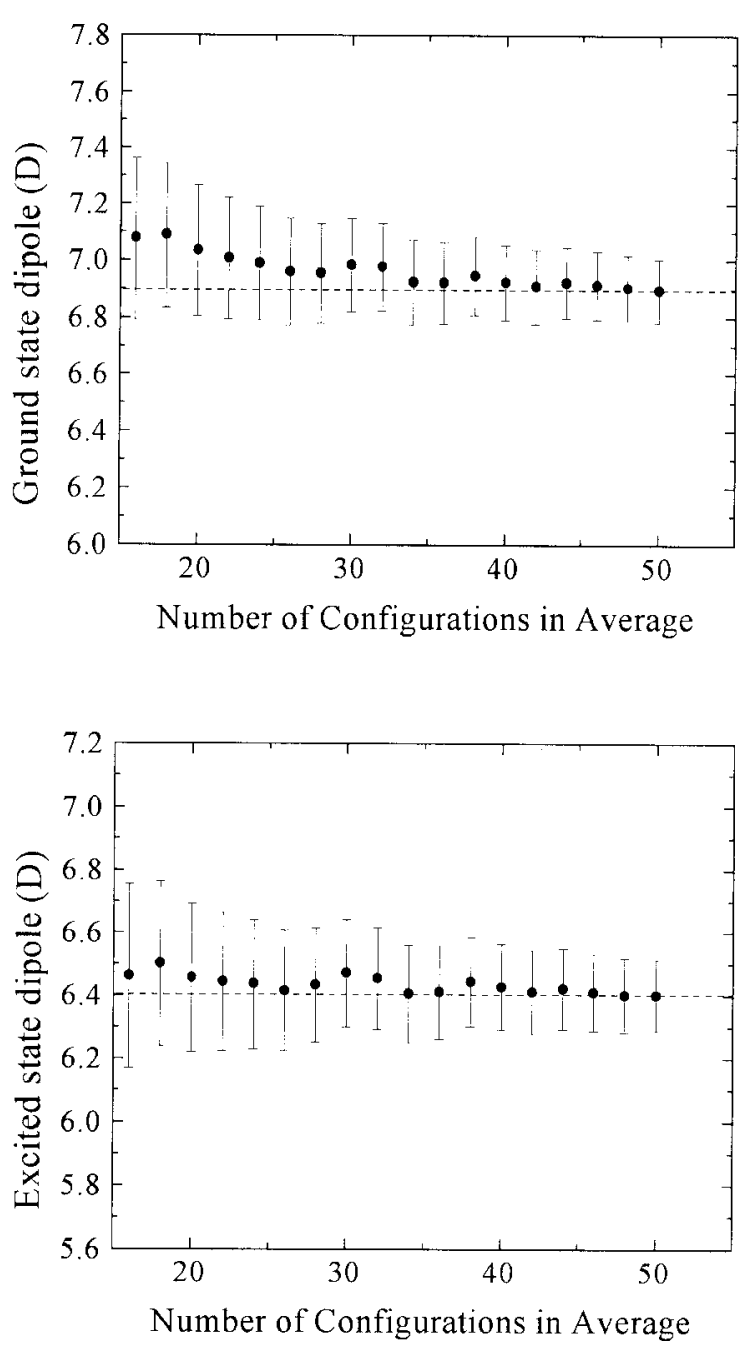

FIGURE 4. Convergence of the CASSCF/6-31G( $(d, p)$ calculated dipole moments for the $1 \mathrm{H}-\mathrm{BZT}+247$ SPC structures. Bars represent the statistical errors of the averages.

averages of the $\mathrm{QM}$ calculations were then taken over 50 configurations. Figure 4 shows the systematic convergence of our results for the ground- and excited-state dipole moments obtained with the CASSCF in the $1+247$ SPC model. As can be seen, the convergence is fast and the use of only $\sim 35$ configurations is sufficient to give statistically converged result. Similar convergence has been obtained in other previous applications [30, 31, 46], including excited-state emission [47]. Our best results here give sizable increases in the calculated dipole moments: The ground-state dipole moment increases by $2.89 \pm 0.10 \mathrm{D}$ and the excited state by $2.75 \pm 0.11 \mathrm{D}$, showing strong electronic polarizations in both states.

\section{SOLVATOCHROMISM}

As direct observations of individual dipole moments in solution are not possible, the simplest way to assess the accuracy of the calculated dipole moments is using the observed solvatochromic shift. 1H-BZT is characterized by a strong absorption transition in the region of $280 \mathrm{~nm}[13,20]$. This transition is seen to shift toward the low wavelength in solvents of increased polarity [20]. In relatively low-polar solvents such as iso-octane the transition maximum is seen at $279 \mathrm{~nm}$. For ethanol the transition changes to $275 \mathrm{~nm}$, leading to a blue shift of $\sim 520 \mathrm{~cm}^{-1}$. An estimate of the gas-to-water shift would give $\sim 700 \mathrm{~cm}^{-1}$ [20]. The blue shift of the $\pi \rightarrow \pi^{*}$ is in qualitative agreement with the decrease of the dipole moment upon excitation. In the gas phase, this decrease amounts to $0.35 \mathrm{D}$. In water solution it changes to $0.49 \mathrm{D}$. The calculated dipole moments can be used to obtain theoretically the solvatochromic shift and the result confronted with the experimental blue shift. One possibility for obtaining the theoretical shift is by using the wellknown Lippert or Bakhshiev relation [48]. A disadvantage is that these relations cannot distinguish the sign (blue or red) of the shift as they depend on the square of the difference in dipole moments. A better relation has been derived by Karelson and Zerner [9] on the basis of the reaction field theory. Accordingly, the shift is given by [9]

$$
\begin{aligned}
\Delta \bar{\nu}=22679 \frac{d}{M M}[ & \frac{\varepsilon-1}{2 \varepsilon+1}\left(\mu_{g} \mu_{g}-\mu_{g} \mu_{e}\right) \\
& \left.+\frac{\eta^{2}-1}{2 \eta^{2}+1}\left(\mu_{g} \mu_{e}-\mu_{e} \mu_{e}\right)\right],
\end{aligned}
$$

where $\mu_{g}$ is the ground-state dipole moment, $\mu_{e}$ is the excited-state dipole moment, $\varepsilon$ is the dielectric constant of the medium $(\varepsilon=80), \eta$ is the index of refraction of the medium $(\eta=1.33), d$ is the density of the solute $\left(d=1.36 \mathrm{~g} / \mathrm{cm}^{3}\right)$, and $M M$ is the molar mass of the solute $(M M=119 \mathrm{amu})$. Using the above relation, we obtained a theoretical solvatochromic blue shift of $570 \mathrm{~cm}^{-1}$, in good agreement with the inferred experimental result of $\sim 700 \mathrm{~cm}^{-1}$. Using the results for the first solvation shell gives the value of $420 \mathrm{~cm}^{-1}$ for the shift (see Table II), a value that seems too low compared to experiment. The inclusion of the water molecules in the outer solvation shells is important to get more reliable values for the total relative polarization of the ground and excited states. As seen in Table II, in 
this respect the excited-state dipole moment is more sensitive to the outer water molecules. Incidentally, using the gas-phase result for the dipole moments implies a blue shift of $230 \mathrm{~cm}^{-1}$. This suggests that the use of the gas-phase values of the dipole moments is useful for the qualitative understanding of the shift but the polarization of the solvent is required to achieve quantitative agreement.

\section{Summary and Conclusions}

The electronic polarization of $1 \mathrm{H}-\mathrm{BZT}$ in water has been calculated using a sequential Monte Carlo quantum mechanics procedure. The structure of the solution of $1 \mathrm{H}-\mathrm{BZT}$ in water has been generated by classic Monte Carlo simulation and subsequently these structures were submitted to ab initio CASSCF calculations of the ground- and first excitedstate dipole moments. Fifty statistically uncorrelated configurations of the Monte Carlo simulation were selected on the basis of the statistical correlation interval obtained from the autocorrelation function of the energy and used in the quantum mechanical calculations to obtain statistically converged results. The $1 \mathrm{H}-\mathrm{BZT}$ suffers a considerable polarization, leading to an increase of the dipole moment by as much as $2.89 \pm 0.10 \mathrm{D}$ for the ground state and $2.75 \pm 0.11 \mathrm{D}$ for the excited state, corresponding to total dipole moments of $6.89 \pm 0.10$ and $6.40 \pm 0.11 \mathrm{D}$, respectively. To validate these numerical results the solvatochromic blue shift of the $\pi \rightarrow \pi^{*}$ excited state was obtained and found to be in good agreement with the experimental observation.

\section{ACKNOWLEDGMENTS}

This work has been partially supported by $\mathrm{CNPq}$ and FAPESP (Brazil), CENAPAD-SP, and LCCAUSP.

\section{References}

1. Onsager, L. J Am Chem Soc 1939, 58, 1486.

2. Gao, J.; Xia, X. Science 1992, 258, 631.

3. Cramer, C. J.; Truhlar, D. G. Science 1992, 256, 213.

4. Leach, A. R. Molecular Modelling. Principles and Applications, $2^{\text {nd }}$ ed.; Prentice-Hall: Englewood Cliffs, NJ, 2001; chap. 4.

5. Silvestrelli, P. L.; Parrinelo, M. Phys Rev Lett 1999, 82, 3308.

6. Tu, Y.; Laaksonen, A. Chem Phys Lett 2000, 329, 283.
7. Poulsen, T. D.; Ogilby, P. R.; Mikkelsen, K. V. J Chem Phys 2002, 116, 3730.

8. Coutinho, K.; Guedes, R. C.; Costa Cabral, B. J.; Canuto, S. Chem Phys Lett 2003, 369, 345, and references therein.

9. Karelson, M.; Zerner, M. C. J Phys Chem 1992, 96, 6949.

10. Liptay, W. In: Sinanoglu, O., ed. Modern Quantum Chemistry, Part II; Academic Press: New York, 1966; p 173.

11. Tomás, F.; Abboud, J. L. M.; Laynez, J.; Notario, R.; Santos, L.; Nilsson, S. O.; Catalán, J.; Claramunt, R. M.; Elguero, J. J Am Chem Soc 1989, 111, 7348.

12. Catalán, J.; Pérez, P.; Elguero, J. J Org Chem 1993, 58, 5276.

13. Tomás, F.; Catalán, J.; Pérez, P.; Elguero, J. J Org Chem 1994, 59, 2799.

14. Kiszka, M.; Dunkin, I. R.; Gebicki, J.; Wang, H.; Wirz, J. J Chem Soc Perkins Trans 2000, 22, 2420.

15. Katritzky, A.; Yannakopoulou, K.; Anders, E.; Stevens, J.; Szafran, M. J Org Chem 1990, 55, 5683.

16. Velino, B.; Cané, E.; Gagliardi, L.; Trombetti, A.; Caminatti, W. J Mol Spectrosc 1993, 161, 136.

17. Roth, W.; Spangenberg, S.; Janzen, C.; Westphal, A.; Schmitt, M. Chem Phys 1999, 248, 17.

18. Schmitt, M.; Plützer, C.; Kleinermanns, K. Phys Chem Chem Phys 2001, 3, 4218.

19. Wofford, D. S.; Forkey, D. M.; Russell, J. G. J Org Chem 1982, 47,5132 .

20. Fagel, J. E. Jr.; Ewing, G. W. J Am Chem Soc 1951, 73, 4360.

21. Coutinho, K.; Canuto, S. Adv Quantum Chem 1997, 28, 90.

22. Canuto, S.; Coutinho, K. Int J Quantum Chem 2000, 77, 192.

23. Roos, B. O. In: Lawley, K. P., ed. Advances in Chemical Physics; Ab Initio Methods in Quantum Chemistry-II; John Wiley \& Sons: Chichester, UK, 1987; pp 399-445.

24. Allen, M. P.; Tildesley, D. J. Computer Simulation of Liquids; Oxford University Press: Oxford, UK, 1987.

25. Berendsen, H. J. C.; Postma, J. P. M.; van Gunsteren, W. F. In: Pullman, B., ed. Intermolecular Forces; Reidel: Dordrecht, The Netherlands, 1981; p 331.

26. Jorgensen, W. L.; Briggs, J. M.; Contreras, M. L. J Phys Chem 1990, 94, 1683; Jorgensen, W. L.; Severance, D. L. J Am Chem Soc 1990, 112, 4768.

27. Werner, H. J.; Knowles, P. J.; with contributions from Almlöf, J.; Amos, R. D.; Berning, A.; Cooper, D. L.; Deegen, M. L. O.; Dobbyn, A. J.; Eckert, F.; Elbert, S. T.; Hampel, C.; Lindh, R.; Loyd, A. W.; Meyer, W.; Niklass, A.; Peterson, K.; Pitzer, R.; Stone, A. J.; Taylor, P. R.; Mura, M. E.; Pulay, P.; Shültz, M.; Stoll, H.; Thorsteinsson, T. MOLPRO, Stuttgart University, Stuttgart (2000).

28. Breneman, C. M.; Wiberg, K. B. J Comput Chem 1990, 11, 361.

29. Escande, A.; Galigné, J. L.; Lapasset, J. Acta Crystallogr B 1974, 30, 1490.

30. Canuto, S.; Coutinho, K.; Trzesniak, D. Adv Quantum Chem 2002, 41, 161.

31. Malaspina, T.; Coutinho, K.; Canuto, S. J Chem Phys 2002, 117, 1692.

32. Coutinho, K.; Canuto, S.; Zerner, M. C. J Chem Phys 2000, 112, 9874.

33. Krätschmer, R.; Binder, K.; Stauffer, D. J Stat Phys 1976, 15, 267. 
34. Roos, B. O.; Fülscher, M. P.; Malmqvist, P.-Å.; Merchán, M.; Serrano-Andrés, L. In: Langhoff, S. R., ed. Quantum Mechanical Electronic Structure Calculations with Chemical Accuracy; Kluwer Academic: Dordrecht, The Netherlands, 1995; p 357.

35. Borin, A. C.; Serrano-Andrés, L.; Fülsher, M. P.; Roos, B. O. J Phys Chem A 1999, 103, 1838.

36. Borin, A. C.; Serrano-Andrés, L. Chem Phys 2000, 262, 253.

37. Serrano-Andrés, L.; Borin, A. C. Chem Phys 2000, 262, 267.

38. Serrano-Andrés, L.; Merchán, M.; Borin, A. C.; Starling, J. Int J Quantum Chem 2001, 84, 181.

39. Roos, B. O.; Andersson, K.; Fülscher, M. P.; Malmqvist, P.-Å.; Serrano-Andrés, L.; Pierloot, K.; Merchán, M. In: Prigogine, I.; Rice, S. A., eds. Advances in Chemical Physics: New Methods in Computational Quantum Mechanics; John Wiley \& Sons: New York, 1996; pp 219-331.

40. Roos, B. O.; Andersson, K.; Fülscher, M. P.; Serrano-Andrés, L.; Pierloot, K.; Merchán, M.; Molina, V. J Mol Struct Theochem 1996, 388, 257.

41. Roos, B. O.; Andersson, K. Chem Phys Lett 1995, 245, 215.
42. Andersson, K.; Barysz, M.; Bernhardsson, A.; Blomberg, M. R. A.; Cooper, D. L.; Fleig, T.; Fülscher, M. P.; de Graff, C.; Hess, B. A.; Karlström, G.; Lindh, R.; Malmqvist, P.-Å.; Neogrády, P.; Olsen, J.; Roos, B. O.; Sadlej, A. J.; Schütz, M.; Schimmelpfennig, B.; Seijo, L.; Serrano-Andrés, L.; Siegbahn, P. E. M.; Stålring, J.; Thorsteinsson, T.; Veryazov, V.; Widmark, P.-O. MOLCAS, version 5; Lund University: Lund, Sweden, 2000.

43. Widmark, P.-O.; Malmqvist, P.-Å.; Roos, B. O. Theor Chim Acta 1990, 77, 291.

44. Coutinho, K.; Canuto, S. DICE, version 2.8; University of São Paulo: São Paulo, 2000.

45. Coutinho, K.; Oliveira, M. J.; Canuto, S. Int J Quantum Chem 1998, 66, 249.

46. Rocha, W. R.; Martins, V. M.; Coutinho, K.; Canuto, S. Theor Chem Acc 2002, 108, 31.

47. Coutinho, K.; Canuto, S. J Chem Phys 2000, 113, 9132.

48. Lippert, Z. Elektrochem 1957, 61, 962; Bakhshiev, N.G. Opt Spektrosk 1964, 16, 821. 